\title{
AN EXTENSION OF A THEOREM OF DOMAR ON INVARIANT SUBSPACES
}

\author{
EVA A. GALLARDO-GUTIÉRREZ, JONATHAN R. PARTINGTON, \\ AND DANIEL J. RODRÍGUEZ
}

\begin{abstract}
A remarkable theorem of Domar asserts that the lattice of the invariant subspaces of the right shift semigroup $\left\{S_{\tau}\right\}_{\tau \geq 0}$ in $L^{2}\left(\mathbb{R}_{+}, w(t) d t\right)$ consists of just the "standard invariant subspaces" whenever $w$ is a positive continuous function in $\mathbb{R}_{+}$ such that

(1) $\log w$ is concave in $[c, \infty)$ for some $c \geq 0$,

(2) $\lim _{t \rightarrow \infty} \frac{-\log w(t)}{t}=\infty$, and $\lim _{t \rightarrow \infty} \frac{\log |\log w(t)|-\log t}{\sqrt{\log t}}=\infty$.

We prove an extension of Domar's Theorem to a strictly wider class of weights $w$, answering a question posed by Domar in [6].
\end{abstract}

\section{Introduction and Preliminaries}

A cornerstone in modern function theory concerning the study of invariant subspaces of shift operators is the celebrated Beurling-Lax-Halmos Theorem. In fact, this theorem completely characterizes all such invariant subspaces in terms of inner functions. Specifically, in the classical Hardy space $H^{2}$ consisting of analytic functions $f(z)=\sum_{n \geq 0} a_{n} z^{n}$ in the open unit disc $\mathbb{D}$ of the complex plane $\mathbb{C}$ such that

$$
\|f\|^{2}=\sum_{n=0}^{\infty}\left|a_{n}\right|^{2}<\infty
$$

the shift operator $S$ is defined to be the operator of multiplication by the coordinate function $z$. In 1949, Beurling [2] proved that any shift-invariant subspace has the form $\theta H^{2}$, where $\theta$ is an inner function. Here, an inner function is an analytic function in $\mathbb{D}$ with contractive values, i.e., $|\theta(z)| \leq 1$ for $z \in \mathbb{D}$, such that its boundary values

$$
\theta\left(e^{i t}\right)=\lim _{r \rightarrow 1^{-}} \theta\left(r e^{i t}\right)
$$

(which exist for almost every point $e^{i t}$ with respect to Lebesgue measure on the unit circle) have modulus one for almost all $e^{i t}$. The usefulness of the result is enhanced by the fact that such an inner function $\theta$ can be factorized as product, in principle, of

Date: December 2015.

1991 Mathematics Subject Classification. Primary 47A15.

Key words and phrases. Right-translation invariant subspaces.

Authors are partially supported by Plan Nacional I+D grant no. MTM2013-42105-P. 
two inner functions: one collecting all the zeros of $\theta$ in $\mathbb{D}$ (a Blaschke product) and the other, lacking zeros in $\mathbb{D}$, a singular inner function, i.e., it can be expressed by an explicit integral formula in terms of a singular measure on the unit circle.

In 1959, Lax [10] extended the result to the finite-dimensional vector-valued case stating his result in the Hardy space of the right half-plane $\mathbb{C}_{+}$. Later, Halmos [9] gave an elegant proof for the infinite-dimensional case and finally, Potapov [13] worked out an analogue of the parametrization (in terms of zeros and a singular measure on the circle) of an inner function for the matrix-valued case. We refer the reader to [12, Chapter 3] for details and further references.

Generalizations and applications of this result have been attracting the attention of many operator theorists. One of the most well-known applications, which is commonly known as the Beurling-Lax Theorem, is the characterization of the lattice of the invariant subspaces of $L^{2}\left(\mathbb{R}_{+}\right)$, the space of measurable functions square-integrable over $[0, \infty)$, under the set $\left\{S_{\tau}: \tau \geq 0\right\}$ of all right shifts:

$$
S_{\tau} f(t)= \begin{cases}0 & \text { if } 0 \leq t \leq \tau \\ f(t-\tau) & \text { if } t>\tau .\end{cases}
$$

In order to state it, recall that the Hardy space $H^{2}\left(\mathbb{C}_{+}\right)$, which consists of the functions $F$ analytic on $\mathbb{C}_{+}$with finite norm

$$
\|F\|_{H^{2}\left(\mathbb{C}_{+}\right)}=\left\{\sup _{0<x<\infty} \int_{-\infty}^{\infty}|F(x+i y)|^{2} d y\right\}^{1 / 2},
$$

is isomorphic under the Laplace transform to $L^{2}\left(\mathbb{R}_{+}\right)$. This is the classical Paley-Wiener Theorem (see [14], for instance), and states that to each function $F \in H^{2}\left(\mathbb{C}_{+}\right)$there corresponds a function $f \in L^{2}\left(\mathbb{R}_{+}\right)$such that

$$
F(s)=(\mathcal{L} f)(s):=\int_{0}^{\infty} f(t) e^{-s t} d t, \quad\left(s \in \mathbb{C}_{+}\right),
$$

and

$$
\|F\|_{H^{2}\left(\mathbb{C}_{+}\right)}^{2}=2 \pi \int_{0}^{\infty}|f(t)|^{2} d t .
$$

By mean of the unitary equivalence $\mathcal{L}: L^{2}\left(\mathbb{R}_{+},(2 \pi) d t\right) \rightarrow H^{2}\left(\mathbb{C}_{+}\right)$, the Beurling-Lax Theorem asserts that a closed subspace $\mathcal{M}$ of $L^{2}\left(\mathbb{R}_{+}\right)$is invariant under every right shift $S_{\tau}, \tau \geq 0$, if and only if there exists an inner function $\Theta \in H^{\infty}\left(\mathbb{C}_{+}\right)$such that $\mathcal{L} \mathcal{M}=\Theta H^{2}\left(\mathbb{C}_{+}\right)$. Recall that an inner function $\Theta$ is an analytic function in $\mathbb{C}_{+}$with $|\Theta(z)| \leq 1$ for $z \in \mathbb{C}_{+}$, such that the non-tangential limits exist and are of modulus 1 almost everywhere on the imaginary axis.

A straightforward observation is that the semigroup $\left\{S_{\tau}: \tau \geq 0\right\}$ has a rich lattice of invariant subspaces in $L^{2}\left(\mathbb{R}_{+}\right)$. Nevertheless, if $w$ denotes a positive function in $\mathbb{R}_{+}$and $L^{2}\left(\mathbb{R}_{+}, w(t) d t\right)$ consists of measurable functions in $\mathbb{R}_{+}$square-integrable respect to the 
measure $w(t) d t$, then the situation changes drastically and the lattice of the invariant subspaces of $\left\{S_{\tau}: \tau \geq 0\right\}$ consists of just the "standard invariant subspaces",

$$
L^{2}([a, \infty), w(t) d t)=\left\{f \in L^{2}\left(\mathbb{R}_{+}, w(t) d t\right): f(t)=0 \text { for a. e. } 0 \leq t \leq a\right\}, \quad(a>0),
$$

whenever $w$ satisfies:

(1) $w$ is a positive continuous function in $\mathbb{R}_{+} \operatorname{such}$ that $\log w$ is concave in $[c, \infty)$ for some $c \geq 0$.

(2) $\lim _{t \rightarrow \infty} \frac{-\log w(t)}{t}=\infty$.

(3) $\lim _{t \rightarrow \infty} \frac{\log |\log w(t)|-\log t}{\sqrt{\log t}}=\infty$.

Actually, this is the content of a remarkable theorem proved by Domar [5] in the eighties. The proof of Domar's Theorem is lengthy and it is based on transferring the problem to a problem in complex function theory and using a key lemma about the growth restriction for subharmonic functions in finitely connected regions in $\mathbb{C}$ (see [6] for a survey of the techniques used in [5]).

Assumption (1) on $w$ seems to play an important role in proving the estimates of the growth of the subharmonic functions in Domar's Theorem. Indeed, such is the case that in [6] Domar poses the question about the nature of such hypothesis on $w$, asking specifically:

Domar's Problem 3. How relevant is the concavity assumption on $\log w$ ?

The aim of this work is to extend Domar's Theorem to a wider class of functions $w$ not necessarily satisfying the condition that $\log w$ is concave in $[c, \infty)$ for any $c \geq 0$, and therefore to provide an answer to Domar's question in some sense. Indeed, we show an example of a continuous weight $w$ satisfying the hypotheses of our main result (Theorem 2.1) although it fails the log-concavity assumption in Domar's Theorem in a strong way, that is, for any continuous weight $\tilde{w}$ inducing an equivalent norm to that one in $L^{2}\left(\mathbb{R}_{+}, w(t) d t\right)$, the function $\log \tilde{w}$ is no longer concave (see Example 2.2, Section 2).

On the other hand, let us point out that while assumption (2) is necessary for unicellularity (all the invariant subspaces are ordered by inclusion); a result of Nikolskii [11] implies that no condition on the magnitude of $-t^{-1} \log w(t)$ at infinity suffices for unicellularity. Note that all the invariant subspaces for $\left\{S_{\tau}\right\}_{\tau \geq 0}$ in $L^{2}\left(\mathbb{R}_{+}, w(t) d t\right)$ are standard if and only if the invariant subspaces for $\left\{S_{\tau}\right\}_{\tau \geq 0}$ in $L^{2}\left(\mathbb{R}_{+}, w(t) d t\right)$ are ordered by inclusion. Hence, to obtain unicellularity, a growth condition on $-t^{-1} \log w(t)$ at infinity has to be combined with a demand of certain regularity of $w$. In this sense, let us also remark that a result of Borichev shows that in the soft topology one can drop off condition (3) in Domar's Theorem (see [3, Theorem 1.6]). Finally, a list of magnitudes of growth which suffice to obtain unicellularity for $\left\{S_{\tau}\right\}_{\tau \geq 0}$ acting boundedly on different weighted Lebesgue spaces with references and discussions is given in [7]. 
The rest of the manuscript is organized as follows. In Section 2 we state our Main Theorem and discuss weights not fulfilling Domar's Theorem. In addition, we will consider logarithmically concave sequences in this context and establish the consequences of our main result at this regard.

Finally, in Section 3, we will provide examples of weights satisfying our main result and discuss them in the framework of the existence conditions for unicellularity in the known literature.

\section{Domar's TheOREM FOR A WIDER ClASS OF WEIGHTS}

In [5], as a consequence of Theorem 2, (see also [5, Section 6] for discussions and [4] for related results), Domar proved the following striking result (stated according to our notation):

Domar's Theorem. Let $w$ be a positive continuous function in $\mathbb{R}_{+}$such that $\log w$ is concave in $[c, \infty)$ for some $c \geq 0$. Assume that

$$
\lim _{t \rightarrow \infty}-\frac{\log w(t)}{t}=\infty
$$

and

$$
\lim _{t \rightarrow \infty} \frac{\log |\log w(t)|-\log t}{\sqrt{\log t}}=\infty .
$$

Then all closed invariant subspaces of the right shift translation operators $\left\{S_{\tau}\right\}_{\tau \geq 0}$ on $L^{2}\left(\mathbb{R}_{+}, w(t) d t\right)$ are the "standard invariant subspaces"

$L^{2}([a, \infty), w(t) d t)=\left\{f \in L^{2}\left(\mathbb{R}_{+}, w(t) d t\right): f(t)=0\right.$ for a. $\left.e .0 \leq t \leq a\right\}, \quad(a>0)$.

A word about notation: Domar denotes by $L^{2}\left(\mathbb{R}_{+}, w(t) d t\right)$ the space of measurable functions $f$ in $\mathbb{R}_{+}$such that $f w \in L^{2}\left(\mathbb{R}_{+}\right)$. Note that this is not affecting the assumptions on $w$ in the previous statement since it is enough to consider the positive continuous function $w^{1 / 2}$.

Now, we are in position to state our main result.

Theorem 2.1. Let $w$ be a positive continuous decreasing weight in $\mathbb{R}_{+}$which satisfies:

(H1) There exists a strictly increasing sequence $\left\{t_{n}\right\}_{n \geq 1} \subset \mathbb{R}_{+}, t_{n} \rightarrow \infty$ as $n \rightarrow \infty$, with $\sup _{n}\left(t_{n+1}-t_{n}\right)<\infty$ and such that the sequence

$$
\left\{\left(w\left(t_{n+1}\right) / w\left(t_{n}\right)\right)^{1 /\left(t_{n+1}-t_{n}\right)}\right\}_{n \geq 1}
$$

is monotone decreasing.

(H2) $\lim _{t \rightarrow \infty} \frac{-\log w(t)}{t}=\infty$. 
(H3) $\lim _{t \rightarrow \infty} \frac{\log |\log w(t)|-\log t}{\sqrt{\log t}}=\infty$.

Then all closed invariant subspaces of $\left\{S_{\tau}\right\}_{\tau \geq 0}$ in $L^{2}\left(\mathbb{R}_{+}, w(t) d t\right)$ are standard.

Before proceeding with the proof, it is worth mentioning that Theorem 2.1 deals with a set of less regular weight functions $w$ than Domar's Theorem in the sense that the log-concavity assumption might not be satisfied for any other weight $\tilde{w}$ such that $L^{2}\left(\mathbb{R}_{+}, \tilde{w}(t) d t\right)$ is $L^{2}\left(\mathbb{R}_{+}, w(t) d t\right)$, as it is shown in the next example.

In what follows, a function $f: \mathbb{R} \rightarrow \mathbb{R}_{+}$such that $\log f$ is concave will be said to be log-concave for short.

Example 2.2. Let $w$ be a weight function in $\mathbb{R}_{+}$such that its logarithm is defined as follows: for every $n \geq 0$, let consider

$$
\log w \mid I_{n}= \begin{cases}-n^{2}-n(t-3 n) & \text { if } 3 n \leq t<3 n+1, \\ -n(n+1) & \text { if } 3 n+1 \leq t<3 n+2, \\ -n(n+1)-2(n+1)(t-3 n-2) & \text { if } 3 n+2 \leq t<3 n+5 / 2, \\ -(n+1)^{2} & \text { if } 3 n+5 / 2 \leq t<3 n+3,\end{cases}
$$

where $I_{n}=[3 n, 3 n+3)$ for all $n \geq 0$.

A simple calculation shows that $w$ satisfies the growth conditions (1) and (2) in Domar's Theorem, even though $\log w$ is not concave (see Figure 1(a)). Moreover, there does not exist an equivalent weight $\tilde{w}$ such that the log-concavity assumption is fulfilled. Indeed, without loss of generality, let $\tilde{w}$ be a log-concave function in $\mathbb{R}_{+}$such that the norms in $L^{2}\left(\mathbb{R}_{+}, w(t) d t\right)$ and $L^{2}\left(\mathbb{R}_{+}, \tilde{w}(t) d t\right)$ are equivalent, which implies that for some $a \leq 0$ we have

$$
a+\log w(t) \leq \log \tilde{w}(t) \leq \log w(t), \quad(t \geq 0) .
$$

Roughly speaking, the graph of the function $\log \tilde{w}$ is contained in the strip whose boundaries are the graph of the functions $\log w$ and $\log w+a$. Now, since $\log \tilde{w}$ is concave, for every $0 \leq x<y<z<\infty$ it follows that

$$
\frac{\log \tilde{w}(y)-\log \tilde{w}(x)}{y-x} \geq \frac{\log \tilde{w}(z)-\log \tilde{w}(y)}{z-y} .
$$

Now, for every $n \geq 0$, if we take $x=3 n, y=3 n+1$ and $z=3 n+2$, the above inequality becomes

$$
L_{n}:=\log \tilde{w}(3 n+1)-\log \tilde{w}(3 n) \geq \log \tilde{w}(3 n+1)-\log \tilde{w}(3 n+1):=R_{n} .
$$

Observe that $L_{n}$ is, in fact, less than or equal to $-n-a$, the derivative of the straight line from $(3 n, \log w(3 n)+a)$ to $(3 n+1, \log w(3 n+1))$. On the other hand, $R_{n}$ is greater or equal to $a$, the derivative of the straight line from $(3 n+1, \log w(3 n+1))$ 


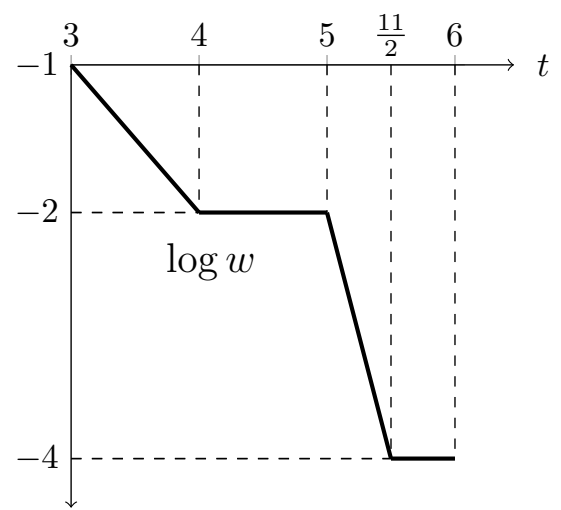

(a) The function $\log w \mid[3,6)$.

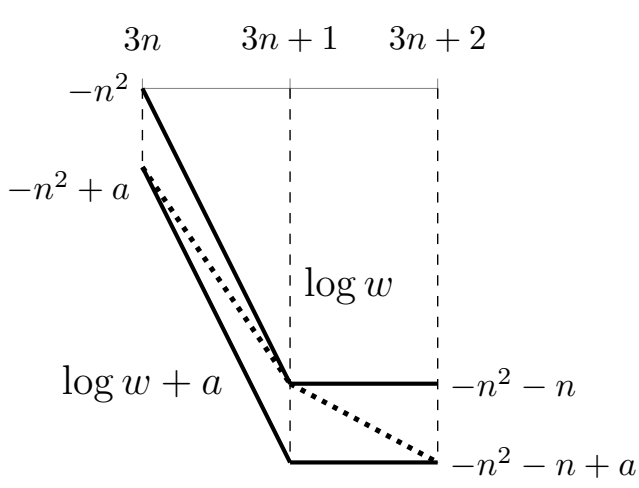

(b) Straight lines.

\section{FiguRE 1}

to $(3 n+2, \log w(3 n+2)+a)$. These straight lines are illustrated by dotted lines in Figure $1(\mathrm{~b})$.

Therefore, the above argument and equation (3) imply that the inequality $-n \geq 2 a$ holds for all $n \geq 0$, which is a contradiction. However, observe that this function $w$ satisfies hypothesis (H1) of the above theorem, just considering the sequence of non-equidistant points $\left\{t_{n}\right\}_{n \geq 1}$ where $t_{2 n-1}=3 n-1$ and $t_{2 n}=3 n$ for all $n \geq 1$.

In light of the aformentioned, as an application of Theorem 2.1, for the weight $w$ defined above all closed invariant subspaces under $\left\{S_{\tau}\right\}_{\tau \geq 0}$ in $L^{2}\left(\mathbb{R}_{+}, w(t) d t\right)$ are standard.

In order to prove Theorem 2.1, we show the following result which is independent, in some sense, of the nature of the weight considered.

Proposition 2.3. Let $w$ be a positive continuous decreasing weight in $\mathbb{R}_{+}$. Let $\mathcal{M}$ be a non-trivial closed invariant subspace of $\left\{S_{\tau}\right\}_{\tau \geq 0}$ in $L^{2}\left(\mathbb{R}_{+}, w(t) d t\right)$. Assume $\mathcal{M}$ contains a non-trivial standard invariant subspace of $\left\{S_{\tau}\right\}_{\tau \geq 0}$. Then $\mathcal{M}$ is standard.

Proof. Assume $\mathcal{M}$ contains the standard invariant subspace $L^{2}([a, \infty), w(t) d t)$, where $a>0$, in $L^{2}\left(\mathbb{R}_{+}, w(t) d t\right)$. Note that the projection $\mathcal{P}$ from $L^{2}\left(\mathbb{R}_{+}, w(t) d t\right)$ onto the space $L^{2}([0, a], w(t) d t)$ takes $\mathcal{M}$ into a closed invariant subspace for $\left\{S_{\tau}\right\}_{\tau \geq 0}$. Since $L^{2}([0, a], w(t) d t)$ is isomorphic to $L^{2}([0, a], d t)$, which in turns is isomorphic to $L^{2}([0,1], d t)$, we are reduced to proving that any closed invariant subspace for $\left\{S_{\tau}\right\}_{\tau \geq 0}$ in the latter space is standard.

Let $N \subset L^{2}([0,1], d t)$ denote a closed invariant subspace for $\left\{S_{\tau}\right\}_{\tau \geq 0}$. Then $N^{\perp} \subset$ $L^{2}([0,1], d t)$ is invariant under $\left\{S_{\tau}^{*}\right\}_{\tau \geq 0}$, and therefore $\mathcal{L} N^{\perp}=K_{\Theta}:=H^{2}\left(\mathbb{C}_{+}\right) \ominus \Theta H^{2}\left(\mathbb{C}_{+}\right)$ for some $\Theta \in H^{\infty}\left(\mathbb{C}_{+}\right)$inner function. Since $K_{\Theta} \subset K_{e^{-s}}$, it follows that $\Theta(s)=e^{-a s}$, with $0 \leq a \leq 1$, and therefore, $N$ is standard. 
With Proposition 2.3 at hand, the strategy to prove Theorem 2.1 will be to construct a positive continuous function $w_{a}$ in $\mathbb{R}_{+}$such that $w(t) \leq w_{a}(t)$ for all $t \in \mathbb{R}_{+}$and satisfying the hypotheses of Domar's Theorem. This construction, accomplished by interpolating the values of the function $w$ at the nodes $\left\{\left(t_{n}, \log w\left(t_{n}\right)\right)\right\}_{n \geq 1}$ from above, would allow us to embed a non-trivial standard invariant subspace in any non-trivial closed invariant subspace of $\left\{S_{\tau}\right\}_{\tau \geq 0}$ in $L^{2}\left(\mathbb{R}_{+}, w(t) d t\right)$.

Proof of Theorem 2.1. Without loss of generality, we may assume that $0<w(t) \leq 1$ for all $t \in \mathbb{R}_{+}$and $M=\sup _{n}\left(t_{n+1}-t_{n}\right) \leq 1$. Otherwise, consider the weight function $\tilde{w}(t)=w(M t)$ for all $t \in \mathbb{R}_{+}$.

Clearly $\tilde{w}$ satisfies the hypothesis (H1), just considering the sequence $\left\{r_{n}\right\}_{n \geq 1}$ where $r_{n}=t_{n} / M$ for all $n \geq 1$. Moreover, since $L^{2}\left(\mathbb{R}_{+}, w(t) d t\right)$ and $L^{2}\left(\mathbb{R}_{+}, \tilde{w}(t) d t\right)$ are isometrically isomorphic by mean of the mapping $f \mapsto M^{1 / 2} f(M t)$, the closed invariant subspaces of the semigroup $\left\{S_{\tau}\right\}_{\tau \geq 0}$ acting on these weighted $L^{2}$ spaces are in one to one correspondence.

Under hypotheses (H1) - (H3), it is possible to construct a weight $w_{a}$ in $\mathbb{R}_{+}$satisfying

(C1) $w(t) \leq w_{a}(t)$ for all $t \in \mathbb{R}_{+}$.

(C2) $w_{a}$ is log-concave in $[c, \infty)$ for some $c \geq 0$.

(C3) $\lim _{t \rightarrow \infty} \frac{-\log w_{a}(t)}{t}=\infty$.

(C4) $\lim _{t \rightarrow \infty} \frac{\log \left|\log w_{a}(t)\right|-\log t}{\sqrt{\log t}}=\infty$.

Indeed, for each $n \geq 0$, let $\Lambda_{n}$ be the linear function defined in the interval $I_{n}=\left[t_{n}, t_{n+1}\right)$, with $I_{0}=\left[0, t_{1}\right)$, for which

$$
\Lambda_{n}\left(t_{n}\right)=\log w\left(t_{n}\right) \text { and } \Lambda_{n}^{-}\left(t_{n+1}\right):=\lim _{t \rightarrow t_{n+1}^{-}} \Lambda_{n}(t)=\log w\left(t_{n+1}\right)
$$

that is

$$
\begin{aligned}
& \Lambda_{0}(t)=\log w(0)+\left(\frac{t}{t_{1}}\right)\left(\log w\left(t_{1}\right)-\log w(0)\right), \quad\left(t \in I_{0}\right), \\
& \Lambda_{n}(t)=\log w\left(t_{n}\right)+\left(\frac{t-t_{n}}{t_{n+1}-t_{n}}\right)\left(\log w\left(t_{n+1}\right)-\log w\left(t_{n}\right)\right), \quad\left(t \in I_{n}\right) .
\end{aligned}
$$

Consider the piecewise function

$$
\Lambda(t)=\Lambda_{0}(t) \chi_{I_{0}}(t)+\sum_{n \geq 1} \Lambda_{n}(t) \chi_{I_{n}}(t), \quad\left(t \in \mathbb{R}_{+}\right)
$$


Note that $\Lambda$ is concave in $\left[t_{1}, \infty\right)$, since $w$ satisfies hypotesis (H1). Now, let define the positive function $w_{a}$ as follows

$$
w_{a}(t)=\left\{\begin{array}{ll}
w(0) & \text { if } 0 \leq t<t_{1}, \\
\exp (\Lambda(t-1)) & \text { if } t \geq t_{1},
\end{array} \quad\left(t \in \mathbb{R}_{+}\right) .\right.
$$

By construction, $\log w_{a}$ is concave in $\left[t_{1}+1, \infty\right)$ and $w(t) \leq w_{a}(t)$ for all $t \in \mathbb{R}_{+}$. Moreover, note that

$$
w_{a}(t+2) \leq w(t), \quad\left(t \geq t_{1}\right)
$$

Indeed, for every $t \geq t_{1}$, there exists $n \geq 1$ (depending on $t$ ) such that $t_{n} \leq t<t_{n+1}$. Hence, since $w$ is decreasing and $t_{n+1}-t_{n} \leq 1$ for all $n \geq 1$, we have

$$
\Lambda(t)=\log w\left(t_{n}\right)+\left(\frac{t-t_{n}}{t_{n+1}-t_{n}}\right)\left(\log w\left(t_{n+1}\right)-\log w\left(t_{n}\right)\right) \leq \log w\left(t_{n}\right) \leq \log w(t-1) .
$$

From here, (5) follows. On the other hand, since $\log w_{a}(t) \leq \log w(t-2)$ for all $t \geq 2+t_{1}$ and $w$ satisfies hypotheses (H2) and (H3), conditions (C3) and (C4) hold.

Now, we are in position to prove that all closed and invariant subspaces of the right shift the semigroup $\left\{S_{\tau}\right\}_{\tau \geq 0}$ in $L^{2}\left(\mathbb{R}_{+}, w(t) d t\right)$ are standard. Condition (C1) implies the inclusion

$$
L^{2}\left(\mathbb{R}_{+}, w_{a}(t) d t\right) \subset L^{2}\left(\mathbb{R}_{+}, w(t) d t\right) .
$$

Let $\mathcal{M} \subset L^{2}\left(\mathbb{R}_{+}, w(t) d t\right)$ be a closed, non-trivial invariant subspace for $\left\{S_{\tau}\right\}_{\tau \geq 0}$. We observe that $S_{2} \mathcal{M} \subset L^{2}\left(\mathbb{R}_{+}, w_{a}(t) d t\right)$, since for every $f \in \mathcal{M}$ we have

$\left\|S_{2} f\right\|_{w_{a}}^{2}=\int_{2}^{\infty}|f(t-2)|^{2} w_{a}(t) d t=\int_{0}^{\infty}|f(t)|^{2} w_{a}(t+2) d t \leq w_{a}(2) \int_{0}^{t_{1}}|f(t)|^{2} d t+\|f\|_{w}^{2}$, using the fact that $w_{a}$ is a decreasing function satisfying equation (5).

Let denote by ${\overline{S_{2} \mathcal{M}}}^{w_{a}}$ the closure of $S_{2} \mathcal{M}$ in $L^{2}\left(\mathbb{R}_{+}, w_{a}(t) d t\right)$, which is an invariant subspace for $\left\{S_{\tau}\right\}_{\tau \geq 0}$ and $w_{a}$ satisfies conditions $(\mathrm{C} 1)-(\mathrm{C} 4)$. Hence, by Domar's Theorem, there exists $c_{a} \in \mathbb{R}_{+} \cup\{\infty\}$ such that

$$
{\overline{S_{2} \mathcal{M}}}^{w_{a}}=L^{2}\left(\left[c_{a}, \infty\right), w_{a}(t) d t\right) .
$$

Observe that $c_{a}<\infty$, since $\mathcal{M} \neq\{0\}$. Clearly $S_{2} \mathcal{M} \subseteq{\overline{S_{2} \mathcal{M}}}^{w_{a}}$ and we have

$$
S_{2} \mathcal{M} \subseteq L^{2}\left(\left[c_{a}, \infty\right), w(t) d t\right) .
$$

Moreover, the density of $L^{2}\left(\left[c_{a}, \infty\right), w_{a}(t) d t\right)$ in $L^{2}\left(\left[c_{a}, \infty\right), w(t) d t\right)$ and the chain of inclusions ${\overline{S_{2} \mathcal{M}}}^{w_{a}} \subset{\overline{S_{2} \mathcal{M}}}^{w} \subset \mathcal{M}$ implies that

$$
L^{2}\left(\left[c_{a}, \infty\right), w(t) d t\right) \subseteq \mathcal{M} .
$$


In addition, $c_{a}>0$ since $\mathcal{M}$ is proper. Thus, $\mathcal{M}$ contains a proper standard invariant subspace of $\left\{S_{\tau}\right\}_{\tau \geq 0}$ in $L^{2}\left(\mathbb{R}_{+}, w(t) d t\right)$, and by Proposition $2.3, \mathcal{M}$ is standard, which concludes the proof of Theorem 2.1.

Remark 2.4. In the above theorem, we can assume without loss of generality that $\lim \sup _{n}\left(t_{n}-t_{n-1}\right)>0$. Otherwise, we may pass to a subsequence $\left\{s_{n}\right\}_{n \geq 1} \subset\left\{t_{n}\right\}_{n \geq 1}$, $s_{n} \rightarrow \infty$ as $n \rightarrow \infty$, with $\delta<\limsup _{n}\left(s_{n}-s_{n-1}\right)<\infty$ for some $\delta>0$ such that the sequence $\left\{\left(w\left(s_{n+1}\right) / w\left(s_{n}\right)\right)^{1 /\left(s_{n+1}-s_{n}\right)}\right\}_{n \geq 1}$ is monotone decreasing.

Remark 2.5. The construction of the weight function $w_{a}$ in Theorem 2.1 is not trivial in the sense that the inclusion in equation (6) is proper. Suppose, on the contrary, that $L^{2}\left(\mathbb{R}_{+}, w_{a}(t) d t\right)=L^{2}\left(\mathbb{R}_{+}, w(t) d t\right)$. For the sequence $\left\{t_{n}\right\}_{n \geq 1}$, we may assume that

$$
\sup _{n}\left(t_{n+1}-t_{n}\right) \leq 1 \text { and } \limsup _{n}\left(t_{n+1}-t_{n}\right)>\delta \text { for some } \delta>0 .
$$

Observe that, for every $n \geq 0$, we have $w(t+1) \leq w\left(t_{n+1}\right)$ for all $t \in I_{n}=\left[t_{n}, t_{n+1}\right)$. From the definition of the weight function $w_{a}$, it follows that the inequality

$$
\frac{w(t+1)}{w_{a}(t+1)} \leq\left(\frac{w\left(t_{n+1}\right)}{w\left(t_{n}\right)}\right)^{\eta(t)} \quad \text { with } \quad \frac{\delta / 2}{t_{n+1}-t_{n}} \leq \eta(t)=\frac{t_{n+1}-t}{t_{n+1}-t_{n}}
$$

holds for all $t \in I_{n}, t_{n+1}-t \geq \delta / 2$, with $t_{n+1}-t_{n}>\delta$. Combining this with the fact that the sequence $\left\{\left(w\left(t_{n+1}\right) / w\left(t_{n}\right)\right)^{1 /\left(t_{n+1}-t_{n}\right)}\right\}_{n \geq 1}$ is decreasing to zero, we have

$$
\liminf \frac{w(t+1)}{w_{a}(t+1)}=0 .
$$

This contradiction proves that the norms in $L^{2}\left(\mathbb{R}_{+}, w(t) d t\right)$ and $L^{2}\left(\mathbb{R}_{+}, w_{a}(t) d t\right)$ are not equivalent.

Recall that a sequence of positive real numbers $\left\{a_{n}\right\}_{n \geq 1}$ is said to be logarithmically concave if $a_{n}^{2} \geq a_{n-1} a_{n+1}$ holds for all $n \geq 2$. If the strictly increasing sequence $\left\{t_{n}\right\}_{n \geq 1}$ in Theorem 2.1 consists of equidistant points, then hypothesis (H1) becomes

$\left(\mathrm{H}^{\prime}\right)\{w(a n+b)\}_{n \geq 1}$ is logarithmically concave for some $a, b>0$.

We will discuss more about this in Subsection 2.1. Nevertheless, let us point out a corollary which follows for $a=1$ and $b=0$.

Corollary 2.6. Let $w$ be a positive continuous decreasing weight in $\mathbb{R}_{+}$satisfying:

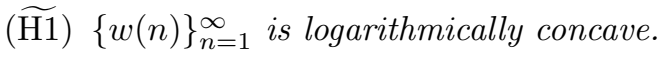

( $\widetilde{\mathrm{H} 2}) \lim _{t \rightarrow \infty} \frac{-\log w(t)}{t}=\infty$.

( $\widetilde{\mathrm{H} 3}) \lim _{t \rightarrow \infty} \frac{\log |\log w(t)|-\log t}{\sqrt{\log t}}=\infty$.

Then all closed invariant subspaces of $\left\{S_{\tau}\right\}_{\tau \geq 0}$ in $L^{2}\left(\mathbb{R}_{+}, w(t) d t\right)$ are standard. 
Remark 2.7. In some particular cases, the function $\Lambda$ defined by equation (4) in Theorem 2.1 coincides with the least concave majorant of $\log w$, as it can be seen for the quasi-concave function in Example 2.2, just considering the sequence $\left\{t_{n}\right\}_{n \geq 1}$ where $t_{2 n-1}=3 n-1$ and $t_{2 n}=3 n$ for all $n \geq 1$.

For the authors, a positive function $f$ on $\mathbb{R}_{+}$is quasi-concave if $f\left(\lambda t_{1}+(1-\lambda) t_{2}\right) \geq$ $\min \left\{f\left(t_{1}\right), f\left(t_{2}\right)\right\}$ holds for every $t_{1}, t_{2} \in \mathbb{R}_{+}$and $0 \leq \lambda \leq 1$. It is worth to mention that there exist other definitions for the notion of quasi-concavity (see [1, Definition 2.5.6], for instance), even though they do not fit in the framework of Domar's Theorem.

2.1. On sequences of nodes with uniformly bounded jumps. In this subsection, we consider logarithmically concave sequences in the context of Domar's Theorem, establishing some consequences of Theorem 2.1.

Regarding Corollary 2.6, it is worth mentioning that when $\left\{t_{n}\right\}_{n \geq 1}$ is an increasing sequence consisting of equidistant points with $t_{n} \rightarrow \infty$ as $n \rightarrow \infty$, the condition

$$
\left(\mathrm{H} 1^{\prime \prime}\right)\left\{w\left(t_{n}\right)\right\}_{n \geq 1} \text { is logarithmically concave, }
$$

implies the existence of a log-concave function $W$ in $\mathbb{R}_{+}$such that

$$
W(t+a) \leq w(t) \leq W(t), \quad(t \geq A), \quad(A \geq 0) .
$$

For the sake of simplicity, we prove such an statement whenever $t_{n}=n$ for all $n \geq 1$.

Proposition 2.8. If $w$ is a positive continuos decreasing weight in $\mathbb{R}_{+}$such that $\{w(n)\}_{n \geq 1}$ is logarithmically concave, then there exists a log-concave function $W$ in $\mathbb{R}_{+}$satisfying equation (8).

Proof. Without loss of generality, we may suppose that $0<w(t) \leq 1$ for all $t \in \mathbb{R}_{+}$and $\log w$ is not concave. For every $n \geq 0$, let $\Lambda_{n}$ be the linear function defined in the interval $[n, n+1)$ for which

$$
\Lambda_{n}(n)=\log w(n) \quad \text { and } \quad \Lambda_{n}^{-}(n+1):=\lim _{t \rightarrow(n+1)^{-}} \Lambda_{n}(t)=\log w(n+1),
$$

that is

$$
\Lambda_{n}(t)=\log w(n)+(t-n)(\log w(n+1)-\log w(n)), \quad(n \leq t<n+1) .
$$

Consider the piecewise function

$$
\Lambda(t)=\sum_{n \geq 0} \Lambda_{n}(t) \chi_{[n, n+1)}(t), \quad\left(t \in \mathbb{R}_{+}\right)
$$

Note that $\Lambda$ is concave in $[1, \infty)$, since $\{w(n)\}_{n \geq 1}$ is logarithmically concave. Let us define the positive function $W$ as follows

$$
W(t)=\left\{\begin{array}{ll}
w(0) & \text { if } 0 \leq t<1, \\
\exp (\Lambda(t-1)) & \text { if } t \geq 1,
\end{array} \quad\left(t \in \mathbb{R}_{+}\right) .\right.
$$


By contruction, the function $\log W$ is concave in $[2, \infty)$. Moreover, since $w$ is decreasing we have

$$
W(t+2)=\exp (\Lambda(t+1)) \leq w(t) \leq W(t), \quad(t \geq 1) .
$$

The converse of the above result is not true, in general, as it is shown in the next example.

Example 2.9. Consider the weight function $w$ in $\mathbb{R}_{+}$such that

$$
\log w \mid\left[a_{n}-2, a_{n}+n\right)=\left\{\begin{array}{ll}
-a_{n} & \text { if } a_{n}-2 \leq t<a_{n}, \\
-a_{n}-\frac{(n+2)}{n}\left(t-a_{n}\right) & \text { if } a_{n} \leq t<a_{n}+n,
\end{array} \quad(n \geq 1),\right.
$$

where $a_{n}=\left(n^{2}+3 n\right) / 2$ for all $n \geq 1$. The function $\log w$ is illustrated in Figure 2 .

Observe that the function $w$ is well-defined, since $a_{n}+n=a_{n+1}-2$ for all $n \geq 1$.

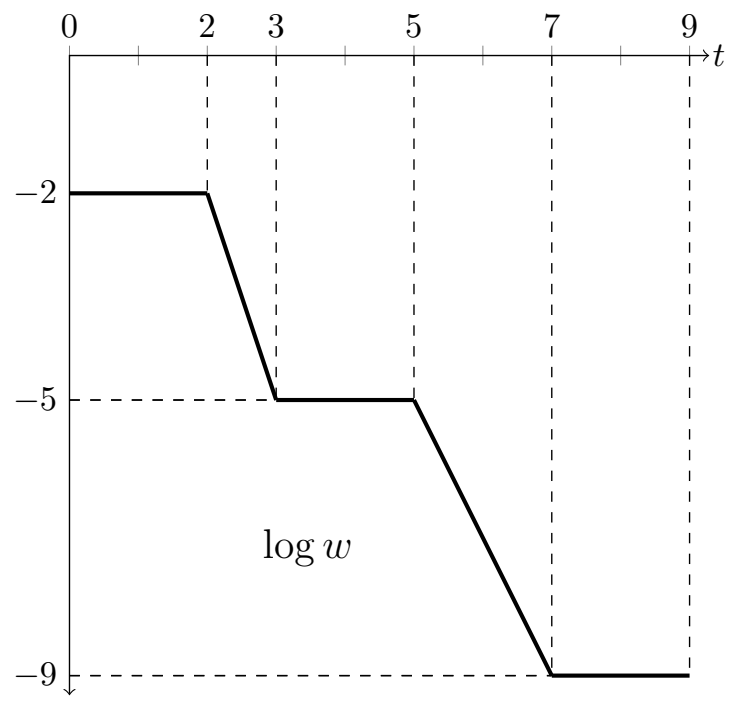

Figure 2. The function $\log w \mid[0,9)$.

Moreover, $-t-2 \leq \log w(t) \leq-t$ for all $t \geq 0$ and, on the intervals of length $n$, we have

$$
(\log w)^{\prime} \mid\left[a_{n}, a_{n}+n\right)=-(n+2) / n \nearrow-1, \quad \text { as } n \rightarrow \infty .
$$

Roughly speaking, $\log w$ and $-t$ are getting closer on the intervals of length $n$.

Without loss of generality, we may assume that $\left\{t_{k}\right\}_{k \geq 1}$ is a strictly increasing sequence of equidistant points, $t_{k} \rightarrow \infty$, such that $d=t_{2}-t_{1}>2$. For $n$ large enough, let $k(n)$ be the positive integer such that

$$
t_{k(n)} \in\left[a_{n}, a_{n}+n\right) \quad, \quad t_{k(n)}+d \in\left(a_{n+1}-2, a_{n+1}\right) \quad \text { and } \quad t_{k(n)}+2 d \in\left[a_{n+1}, a_{n+1}+n+1\right)
$$


where

$$
\begin{aligned}
\frac{w\left(t_{k(n)}+d\right)}{w\left(t_{k(n)}\right)} & =\exp \left(-a_{n(k)+1}+a_{n(k)}+\frac{n(k)+2}{n(k)}\left(t_{k}-a_{n(k)}\right)\right), \\
\frac{w\left(t_{k(n)}+2 d\right)}{w\left(t_{k(n)}+d\right)} & =\exp \left(-\frac{n(k)+3}{n(k)+1}\left(t_{k}+2 d-a_{n(k)+1}\right)\right) .
\end{aligned}
$$

Observe that $k(n) \rightarrow \infty$ as $n \rightarrow \infty$. Assuming $\left\{w\left(t_{k}\right)\right\}_{k \geq 1}$ is logarithmically concave, a simple computation shows that equations (9) and (10) imply that the inequality

$$
\left(\frac{n(k)+3}{n(k)+1}+\frac{n(k)+2}{n(k)}\right)\left(t_{n(k)}-a_{n(k)}\right)+(2 d-n(k)-2)\left(\frac{n(k)+3}{n(k)+1}\right) \geq n(k)+2
$$

holds for $n$ large enough, which is a contradiction since $t_{n(k)}-a_{n(k)}$ behaves like $n(k)$.

However, it is worth mentioning that when $\left\{t_{n}\right\}_{n \geq 1} \subset \mathbb{R}_{+}$is a sequence of nodes with uniformly bounded jumps, that is, a strictly increasing sequence of non-equidistant points such that $t_{n} \rightarrow \infty$ as $n \rightarrow \infty$ with $\sup _{n}\left(t_{n+1}-t_{n}\right)<\infty$, then the condition $\left(\mathrm{H} 1^{\prime \prime}\right)$ does not imply the existence of a log-concave function $W$ in $\mathbb{R}_{+}$satisfying equation (8), even when the growth conditions in Domar's Theorem are fulfilled.

Example 2.10. Let $w$ be a positive continuous decreasing weight in $\mathbb{R}_{+}$such that its logarithm is given by

$\log w \mid I_{n}=\left\{\begin{array}{ll}a_{n}-\left(t-T_{n}\right) n^{2} & \text { if } T_{n} \leq t<T_{n}+1, \\ a_{n}-n^{2}+\left(\frac{t-T_{n}-1}{n+2}\right)\left(a_{n+1}-a_{n}+n^{2}\right) & \text { if } T_{n}+1 \leq t<T_{n+1},\end{array} \quad(n \geq 0)\right.$, where $I_{n}=\left[T_{n}, T_{n+1}\right), T_{n}=n(n+5) / 2$ and $a_{n}=-n^{3}$ for all $n \geq 0$. Observe that $w$ is a continuous piecewise-linear function such that the derivative of $\log w$ behaves, roughly speaking, like

- $(\log w)^{\prime}=-n^{2}$ on an interval of length 1 ,

- $(\log w)^{\prime}=-2 n$ (approx.) on an interval of length $n+2$,

and so on.

For this function $\log w$, we prove the following statements:

(1.) There exists an increasing sequence $\left\{t_{n}\right\}_{n \geq 0} \subset \mathbb{R}_{+}, t_{n} \rightarrow \infty$ as $n \rightarrow \infty$, with $\sup _{n}\left(t_{n+1}-t_{n}\right)<\infty$ such that $\left\{w\left(t_{n}\right)\right\}_{n \geq 0}$ is logarithmically concave.

(2.) There does not exist a concave function $\psi$ in $\mathbb{R}_{+}$and $a>0$ such that

$$
\psi(t+a) \leq \log w(t) \leq \psi(t), \quad(t \geq A), \quad(A \geq 0)
$$

(3.) The function $\log w$ satisfies the following growth conditions

$$
\lim _{t \rightarrow \infty} \frac{-\log w(t)}{t}=\infty \quad \text { and } \quad \lim _{t \rightarrow \infty} \frac{\log |\log w(t)|-\log t}{\sqrt{\log t}}=\infty
$$


Indeed:

(1.) For every $n \geq 0$, let $\left\{t_{j}^{I_{n}}\right\}_{j=1}^{N_{n}}$ be a collection of points in the interval $I_{n}$ with $T_{n}=t_{1}^{I_{n}}<t_{2}^{I_{n}}<\cdots<t_{m_{n}+1}^{I_{n}}=T_{n}+1<\cdots<t_{N_{n}}^{I_{n}}<T_{n+1}, \quad\left(m_{n}, N_{n} \in \mathbb{Z}_{+}, N_{n}<\infty\right)$, such that $\log w\left(t_{j}^{I_{n}}\right)-\log w\left(t_{j-1}^{I_{n}}\right)=-1$ for all $j=2,3, \ldots, N_{n}$. For the subinterval $\left[T_{n}, T_{n}+1\right)$, it is easy to check that $\left|t_{j}^{I_{n}}-t_{j-1}^{I_{n}}\right|<1$ for all $j=2,3, \ldots, m_{n}$. On the other hand, for the subinterval $\left[T_{n}+1, T_{n+1}\right)$, from the definition of the function $\log w$, a simple computation shows that $t_{m_{n}+k}^{I_{n}}$ is given by

$$
t_{m_{n}+k}^{I_{n}}=T_{n}+1+k\left(\frac{n+2}{2 n^{2}+3 n+1}\right), \quad\left(1 \leq k<K_{0} \text { with } b_{n}-n^{2}-K_{0}=b_{n+1}\right) .
$$

In this case, for every $k$ we have $\left|t_{m_{n}+k+1}^{I_{n}}-t_{m_{n}+k}^{I_{n}}\right|=(n+2) /\left(2 n^{2}+3 n+1\right) \leq 1$ for all $n \geq 0$. Observe that, for every $n \geq 1$, consecutive points differ from each other by $1 / n^{2}$ on the interval $\left[T_{n}, T_{n}+1\right)$ and, approximately, by $1 / 2 n$ on the interval $\left[T_{n}+1, T_{n+1}\right)$.

Therefore, from the above procedure, the double indexed sequence $\Lambda=\left\{\left\{t_{j}^{I_{n}}\right\}_{j=1}^{N_{n}}\right\}_{n \geq 0}$ is such that $\left\{\left\{w\left(t_{j}^{I_{n}}\right)\right\}_{j=1}^{N_{n}}\right\}_{n \geq 0}$ is logarithmically concave with $\sup _{j}\left(t_{j}^{I_{n}}-t_{j-1}^{I_{n}}\right) \leq 1$ for all $n \geq 0$.

(2.) For this statement, consider the piecewise linear function

$$
\phi(t)=\sum_{n \geq 0} \phi_{n}(t) \chi_{I_{n}}(t), \quad\left(t \in \mathbb{R}_{+}\right),
$$

where

$$
\phi_{n}(t)=a_{n}+\left(\frac{t-T_{n}}{n+3}\right)\left(a_{n+1}-a_{n}\right), \quad\left(T_{n} \leq t<T_{n+1}\right), \quad(n \geq 0) .
$$

The function $\phi$ defined above is, in fact, the least concave majorant for the function $\log w$, which is obtained interpolating the nodes $\left(T_{n}, a_{n}\right)$ and $\left(T_{n+1}, a_{n+1}\right)$ by a linear function. Now, we show that

$$
\sup _{n}\left|(\log w)^{-1}\left(a_{n}-n^{2}\right)-\phi^{-1}\left(a_{n}-n^{2}\right)\right|=\infty .
$$

Roughly speaking, we prove that there is a sequence of gaps, getting bigger in time, between the functions $\log w$ and $\phi$. From the definition of the function $\log w$, it is clear that $(\log w)^{-1}\left(a_{n}-n^{2}\right)=T_{n}+1$ for all $n \geq 0$.

On the other hand, for every $n \geq 0$, we look for a point $t_{n}^{*} \in I_{n}$ such that

$$
a_{n}-n^{2}=\phi\left(t_{n}^{*}\right)=a_{n}+\left(\frac{t_{n}^{*}-T_{n}}{n+3}\right)\left(a_{n+1}-a_{n}\right) .
$$

Since $a_{n+1}-a_{n}=-3 n^{2}-3 n-1$ for all $n \geq 0$, a simple calculation shows that, for every $n \geq 0$, the point $t_{n}^{*}$ is given by $t_{n}^{*}=T_{n}+n^{2}(n+3) /\left(3 n^{2}+3 n+1\right)$. Note that the point 
$t_{n}^{*} \in I_{n}$, since $t_{n}^{*}-T_{n}<n+3=T_{n+1}-T_{n}$, for all $n \geq 0$. Hence

$$
\left|(\log w)^{-1}\left(a_{n}-n^{2}\right)-\phi^{-1}\left(a_{n}-n^{2}\right)\right|=\frac{n^{2}(n+3)}{3 n^{2}+3 n+1}-1 \rightarrow \infty, \quad \text { as } n \rightarrow \infty .
$$

Due to this fact, we conclude that there does not exist a concave function $\psi$ in $\mathbb{R}_{+}$ satisfying equation (11) for any $a>0$. Otherwise, from the definition of the least concave majorant function $\phi$, ones has $\phi \leq \psi$ and thus $\phi$ would satisfy the chain of inequalities $\phi(t+a) \leq w(t) \leq \phi(t)$ for some $t \geq A$, which is a contradiction.

(3.) Finally, for this statement, the inequality $a_{n+1} \leq \log w(t) \leq a_{n}$ holds for all $t \in I_{n}, n \geq 1$, which implies

$$
\begin{aligned}
&-\frac{\log w(t)}{t} \geq-\frac{a_{n}}{T_{n+1}}=\frac{2 n^{3}}{(n+1)(n+6)} \rightarrow \infty, \text { as } n \rightarrow \infty . \\
& \frac{\log |\log w(t)|-\log t}{\sqrt{\log t}} \geq \frac{\log \left|2 n^{3} /(n+1)(n+6)\right|}{\sqrt{\log (n+1)(n+6)}} \sim \frac{\log n}{\sqrt{2 \log n}} \rightarrow \infty, \quad \text { as } n \rightarrow \infty .
\end{aligned}
$$

We finish this section with the following observation concerning the above example.

Remark 2.11. For the weight function $w$ defined above, using a similar argument to the one in Example 2.2, it can be proved that the log-concavity assumption is not satisfied for any weight function $\tilde{w}$ such that the norms in $L^{2}\left(\mathbb{R}_{+}, w(t) d t\right)$ and $L^{2}\left(\mathbb{R}_{+}, \tilde{w}(t) d t\right)$ are equivalent.

\section{A final Remark on Domar's Weights}

In this section, we provide examples of weight functions in the context of Domar's Theorem. We begin by exhibiting examples of non-standard invariant subspaces in $L^{2}\left(\mathbb{R}_{+}, w(t) d t\right)$, where $w$ is any weight functions $w$ not satisfying condition (2) in Domar's Theorem. Such examples are based on similar ones given by Domar in the sequence space $\ell^{p}(w)$ (see [4, pp. 134]).

Example 3.1. Let $w$ be a positive continuous function on $\mathbb{R}_{+}$such that

$$
\liminf \frac{\log w(t)}{t}>-\infty \text {. }
$$

Let $\alpha$ be a complex number such that its real part $\operatorname{Re}(\alpha)$ is smaller than the left hand side of (12), which implies that

$$
C_{\alpha}=\int_{0}^{\infty} \exp (\operatorname{Re}(\alpha) t) w(t)^{-1} d t
$$

is finite. Consider the non-trivial subspace in $L^{2}\left(\mathbb{R}_{+}, w(t) d t\right)$ given by

$$
\mathcal{M}_{\alpha}=\left\{f \in L^{2}\left(\mathbb{R}_{+}, w\right): \int_{0}^{\infty} f(t) \exp (\alpha t / 2) d t=0\right\} .
$$


Clearly, it is invariant for the semigroup of translation operators $\left\{S_{\tau}\right\}_{\tau \geq 0}$. Moreover, given a sequence $\left\{f_{n}\right\}_{n \geq 1} \subset \mathcal{M}_{\alpha}$ converging to some $f \in L^{2}\left(\mathbb{R}_{+}, w(t) d t\right)$, we have

$$
\begin{aligned}
\left|\int_{0}^{\infty} f(t) \exp (\alpha t / 2) d t\right| & \leq \int_{0}^{\infty}\left|f(t)-f_{n}(t)\right| \exp (\operatorname{Re}(\alpha) t / 2) d t \\
& \leq\left(\int_{0}^{\infty} \exp (\operatorname{Re}(\alpha) t) w(t)^{-1} d t\right)^{1 / 2}\left(\int_{0}^{\infty}\left|f(t)-f_{n}(t)\right|^{2} w(t)\right)^{1 / 2} \\
& =C_{\alpha}^{2}\left\|f-f_{n}\right\|_{w} \rightarrow 0, \quad \text { as } n \rightarrow \infty .
\end{aligned}
$$

Therefore, $\mathcal{M}_{\alpha}$ is a closed invariant subspace for $\left\{S_{\tau}\right\}_{\tau \geq 0}$ in $L^{2}\left(\mathbb{R}_{+}, w(t) d t\right)$. Finally, observe that $\mathcal{M}_{\alpha}$ is a non-standard invariant subspace for $\left\{S_{\tau}\right\}_{\tau \geq 0}$ since, for instance, the function

$$
f(t)=\left(\chi_{[a, b]}-\chi_{[c, d]}\right)(t) \exp (\bar{\alpha} t / 2), \quad\left(t \in \mathbb{R}_{+}\right),
$$

belongs to $\mathcal{M}_{\alpha}$ for some suitable non-negative numbers $0 \leq a<b<c<d<\infty$.

3.1. Star-shaped weights. In the context of Banach algebras, well-behaved sequences of weights $\{w(n)\}_{n \geq 0}$ have played important roles in order to ensure that all closed ideals are standard in $\ell^{1}(w(n))$. More precisely, if $\{w(n)\}_{n \geq 0}$ is a sequence of positive reals such that

$$
\begin{gathered}
w(0)=1 \text { and } 0<w(n) \leq 1 \text { for all } n \in \mathbb{Z}^{+}, \\
w(m+n) \leq w(m) w(n) \text { for all } m, n \in \mathbb{Z}^{+}, \\
\qquad \lim _{n \rightarrow \infty} w(n)^{1 / n}=0,
\end{gathered}
$$

then the subalgebra of the formal power series

$$
\ell^{1}(w(n))=\left\{y=\sum_{n=0}^{\infty} y_{n} z^{n}:\|y\|=\sum_{n=0}^{\infty}\left|y_{n}\right| w(n)<\infty\right\}
$$

is a radical Banach algebra with identity adjoined. The multiplication is given by the usual multiplication of formal power series. Besides $\ell^{1}(w(n))$, there are obvious closed ideals in $\ell^{1}(w(n))$ :

$$
\mathcal{M}_{k}=\left\{\sum_{n=0}^{\infty} y_{n} z^{n} \in \ell^{1}(w(n)): y_{0}=y_{1}=\cdots=y_{k-1}=0\right\}
$$

for $k=1,2, \cdots$ and of course the zero ideal. Such closed ideals are referred to as standard ideals. A theorem of Grabiner [8, Theorem 4.1] states that if $\{w(n)\}_{n \geq 0}$ is a logarithmically concave sequence, then all closed ideals in $\ell^{1}(w(n))$ are standard (see also [17, Theorem 4] for a related result).

A weaker requirement is that the weight sequence $\{w(n)\}_{n \geq 0}$ be star-shaped. Essentially, this means that the region below the graph of $y=\log w$ is illuminated by the origin; equivalently $\left\{w(n)^{1 / n}\right\}_{n \geq 1}$ is a decreasing sequence. In this case also, all closed 
ideals of $\ell^{1}(w(n))$ are standard provided $w(n)^{1 / n}$ is $O\left(1 / n^{a}\right)$ for some $a>0$ (see [16, Corollary 3.6]). A construction of a radical Banach algebra $\ell^{1}(w(n))$ with a non-standard ideal was shown in [15].

In the context of Domar's Theorem discussed in the previous sections, one of the arguments in the proofs has been to interpolate at the corresponding nodes by a log-concave function. So, it seems natural to ask if we are able to interpolate when hypotheses $(\mathrm{H} 1)$ in Theorem 2.1 (or the corresponding ( $\widetilde{\mathrm{H} 1}$ ) in Corollary 2.6) are replaced by the weaker condition of $\{w(n)\}_{n \geq 0}$ being star-shaped.

First, we observe the following.

Proposition 3.2. Let $\left\{a_{n}\right\}_{n \geq 0} \subset \mathbb{R}_{+}$be a logarithmically concave non-zero sequence with $a_{0}=1$. Then the sequence $\left\{a_{n}^{1 / n}\right\}_{n \geq 1}$ decreases. Moreover, if $a_{n+1} / a_{n} \searrow 0$ as $n \rightarrow \infty$, then $\left\{a_{n}\right\}_{n \geq 0}$ is star-shaped.

Proof. Let denote $\beta_{n}=\log a_{n}$ for all $n \geq 0$. It suffices to show that $n \beta_{n+1} \leq(n+1) \beta_{n}$ holds for all $n \geq 0$. Since $\left\{a_{n}\right\}_{n \geq 0}$ is logarithmically concave it follows that

$$
\beta_{n+1} \leq 2 \beta_{n}-\beta_{n-1}, \quad(n \geq 1)
$$

Now, it can be shown inductively that

$$
n \beta_{n+1} \leq(n+1) \beta_{n}, \quad(n \geq 1)
$$

For $n=1$, equations (13) and (14) are the same. Now, assume (14) remains true for some $n_{0} \geq 1$, that is, $n_{0} \beta_{n_{0}+1} \leq\left(n_{0}+1\right) \beta_{n_{0}}$. By (13), it follows that

$$
\beta_{n_{0}+2} \leq 2 \beta_{n_{0}+1}-\beta_{n_{0}} \leq 2 \beta_{n_{0}+1}-\frac{n_{0}}{n_{0}+1} \beta_{n_{0}+1}=\frac{n_{0}+2}{n_{0}+1} \beta_{n_{0}+1} .
$$

So, $\left(n_{0}+1\right) \beta_{n_{0}+2} \leq\left(n_{0}+2\right) \beta_{n_{0}+1}$. Therefore, equation (14) holds for $n_{0}+1$. and $\left\{a_{n}^{1 / n}\right\}_{n \geq 1}$ is a decreasing sequence. To complete the proof, note that $a_{n+1} / a_{n} \searrow 0$ as $n \rightarrow \infty$ implies that $a_{n}^{1 / n} \rightarrow 0$ as $n \rightarrow \infty$.

Example 3.3. Let us remark that not every star-shaped sequence is logarithmically concave. For instance, consider the decreasing sequence $\left\{a_{n}\right\}_{n \geq 0} \subset \mathbb{R}_{+}$given by

$$
a_{0}=1, \quad a_{2 n-1}=\exp \left(-(2 n-1)^{2}\right), \quad a_{2 n}=\exp \left(-(2 n)^{2}+2 n-1\right), \quad(n \geq 1),
$$

A straightforward computation shows that $\left\{a_{n}^{1 / n}\right\}_{n \geq 1}$ decreases to zero but it is not logarithmically concave, since $a_{2 n+1}^{2}<a_{2 n} a_{2 n+2}$ holds for all $n \geq 2$. Hence, this example shows that is not possible to interpolate at the nodes $\left\{\left(n, a_{n}\right)\right\}_{n \geq 1}$ by a log-concave function; as it is carried over in the proof of Proposition 2.8. 
Finally, the next example shows, in the context of Theorem 2.1, the existence of a strictly increasing sequence $\left\{t_{n}\right\}_{n \geq 1} \subset \mathbb{R}_{+}$, with $t_{n} \rightarrow \infty$ as $n \rightarrow \infty$ such that

$$
\left\{\left(w\left(t_{n+1}\right) / w\left(t_{n}\right)\right)^{1 /\left(t_{n+1}-t_{n}\right)}\right\}_{n \geq 1}
$$

decreases but $\left\{w\left(t_{n}\right)\right\}_{n \geq 1}$ is not a star-shaped weight.

Example 3.4. Let us fix $t_{0}, t_{1}, \beta_{0}$ and $\beta_{1}$ real numbers with $\beta_{1}<\beta_{0} \leq 0 \leq t_{0}<t_{1}$. The sequence $\left\{\beta_{n}\right\}_{n \geq 1}$ is defined recursively as follows: take $\beta_{2 n}$ a real number satisfying

$$
\begin{array}{r}
\left(t_{2 n-1}-t_{2 n-2}\right) \frac{\beta_{2 n-1}-\beta_{2 n}}{\beta_{2 n-2}-\beta_{2 n-1}}>1, \\
(2 n-1) \beta_{2 n} \leq 2 n \beta_{2 n-1} .
\end{array}
$$

Choose a positive real number $t_{2 n}>t_{2 n-1}$ such that

$$
\frac{\beta_{2 n}-\beta_{2 n-1}}{t_{2 n}-t_{2 n-1}} \leq \frac{\beta_{2 n-1}-\beta_{2 n-2}}{t_{2 n-1}-t_{2 n-2}},
$$

that is, $t_{2 n}-t_{2 n-1} \leq\left(t_{2 n-1}-t_{2 n-2}\right)\left(\beta_{2 n-1}-\beta_{2 n}\right) /\left(\beta_{2 n-2}-\beta_{2 n-1}\right)$. In order to ensure that $t_{n} \rightarrow \infty$ as $n \rightarrow \infty$, take $t_{2 n}$ such that

$$
1 \leq t_{2 n}-t_{2 n-1} \leq \frac{\beta_{2 n-1}-\beta_{2 n-2}}{t_{2 n-1}-t_{2 n-2}},
$$

which is possible because $\beta_{2 n}$ satisfies inequality (15).

Next, let $\beta_{2 n+1}$ be a real number with $\beta_{2 n} \geq \beta_{2 n+1}$, for which the strict inequality $2 n \beta_{2 n+1}>(2 n+1) \beta_{2 n}$ holds, and choose a positive real number $t_{2 n+1}>t_{2 n}$ such that

$$
\frac{\beta_{2 n}-\beta_{2 n-1}}{t_{2 n}-t_{2 n-1}} \leq \frac{\beta_{2 n-1}-\beta_{2 n-2}}{t_{2 n-1}-t_{2 n-2}},
$$

that is, $t_{2 n+1}-t_{2 n} \leq\left(t_{2 n}-t_{2 n-1}\right)\left(\beta_{2 n}-\beta_{2 n+1}\right) /\left(\beta_{2 n-1}-\beta_{2 n}\right)$.

Now, let $w>0$ be a continuous function in $\mathbb{R}_{+}$such that $\beta_{n}=\log w\left(t_{n}\right)$ for all $n \geq 1$. By construction, we get the sequence $\left\{\left(w\left(t_{n+1}\right) / w\left(t_{n}\right)\right)^{1 /\left(t_{n+1}-t_{n}\right)}\right\}_{n \geq 1}$ decreases for the sequence $\left\{t_{n}\right\}_{n \geq 1}$, with $t_{n} \rightarrow \infty$ as $n \rightarrow \infty$, but $\left\{w\left(t_{n}\right)\right\}_{n \geq 1}$ is not a star-shaped weight, since $w\left(t_{2 n+1}\right)^{1 / 2 n+1}>w\left(t_{2 n}\right)^{1 / 2 n}$ holds for all $n \geq 1$.

\section{ACKNOWLEDGEMENTS}

The third author acknowledges Ministerio de Economía y Competitividad (Spain) the research grant "Ayudas a la movilidad predoctoral para la realización de estancias breves en centros I+D", ref. EEBB-I-14-08134. The first and third authors are grateful to the School of Mathematics at the University of Leeds for the hospitality shown during their stay. 
18 EVA A. GALLARDO-GUTIÉRREZ, JONATHAN R. PARTINGTON, AND DANIEL J. RODRÍGUEZ

\section{REFERENCES}

[1] C. Bennett and R. C. Sharpley, Interpolation of Operators, Academic Press, 1998.

[2] A. Beurling, On two problems concerning linear transformations in Hilbert space, Acta Math., 81 (1949), 239-255.

[3] A. Borichev, The generalized Fourier transform, the Titchmarsh theorem and almost analytic functions, Leningrad Math. J. 1 (1990), no. 4, 825-857.

[4] Y. Domar, Translation invariant subspaces of weighted $\ell^{p}$ and $L^{p}$ spaces, Math. Scand. 49 (1981), 133-144.

[5] Y. Domar, Extensions of the Titchmarsh convolution theorem with application in the theory of invariant subspaces, Proc. London Math. Soc. 46 (1983), 288-300.

[6] Y. Domar, A solution of the translation-invariant subspace problem for weighted $L^{p}$ on $\mathbb{R}, \mathbb{R}^{+}$or $\mathbb{Z}$, Radical Banach algebras and automatic continuity, 214-226, Lecture Notes in Math., 975, Springer, 1983.

[7] Y. Domar, Translation-invariant subspaces of weighted $L^{p}$, Contemp. Math., 91, Amer. Math. Soc., Providece, RI, 1989.

[8] S. Grabiner, Weighted shifts and Banach algebras of power series, Amer. J. Math., 97, (1975), 16-42.

[9] P.R. Halmos, Shifts on Hilbert spaces, J. Reine Angew. Math., 208 (1961), 102-112.

[10] P.D. Lax, Translation invariant subspaces, Acta Math., 101 (1959), 163-178.

[11] N. K. Nikolskii, Unicellularity and non-unicellularity of weighted shift operators, Dokl. Ak. Nauk SSR 172 (1967), 287-290.

[12] J. R. Partington, Linear operators and linear systems, Cambridge University Press, 2004.

[13] V.P. Potapov, The multiplicative structure of J-contractive matrix functions, Amer. Math. Soc. Transl., 15 : 2 (1960), 131-243.

[14] W. Rudin, Real and complex analysis, $3^{\text {rd }}$ edition, McGraw-Hill, New York, 1987.

[15] M. P. Thomas, A non-standard ideal of a radical Banach algebra of power series, Acta Math. 152 (1984), 199-217.

[16] M. P. Thomas, Approximation in the radical algebra $\ell^{1}(w(n))$ when $\{w(n)\}$ is star-shaped, Radical Banach algebras and automatic continuity, Lecture Notes in Math., 975, Springer, 1983.

[17] D. V. Yakubovich, Invariant subspaces of weighted shift operators, J. Sov. Math. 37, 1323-1346 (1987). 
Universidad Complutense de Madrid e iCMAT

Departamento de Análisis Matemático,

Facultad de Ciencias Matemáticas,

Plaza de Ciencias 3

28040, MADRID (SPAIN)

E-mail address: eva.gallardo@mat.ucm.es

School of Mathematics,

UNIVERSITY OF LEEDS,

LEEDS LS2 9JT, U.K.

E-mail address: J.R.Partington@leeds.ac.uk

Departamento de Matemáticas,

Facultad de Ciencias,

Universidad DE Zaragoza

Plaza San Francisco s/N

50009, Zaragoza (SPAIN)

E-mail address: drluis@unizar.es 